\title{
Subspace-MMSE Blind Channel Estimation for Multiuser OFDM with Receiver Diversity
}

\author{
Yu-Yi Cheng Yumin Lee Hsueh-Jyh Li \\ Graduate Institute of Communication Engineering \\ National Taiwan University \\ Taipei 10617, Taiwan
}

\begin{abstract}
In this paper, a receiver algorithm for OFDM with receiver diversity is proposed. The proposed receiver uses a new two-step blind channel estimation algorithm referred to as the subspace-MMSE channel estimator. Minimum mean square error linear diversity combining is then performed based on the estimated channel. Simulation results show that performance gain of $1-3 \mathrm{~dB}$ is achievable by the proposed approach.
\end{abstract}

\section{INTRODUCTION}

Orthogonal frequency division multiplexing (OFDM) [1] is an efficient transmission technique for high-rate wireless data communication systems. In OFDM, a high-rate data stream is transmitted over low-rate parallel subcarriers, thus increasing the symbol duration and reducing the effect of multipath delay-spread. A cyclic prefix (CP) is also introduced in every OFDM symbol to eliminate inter-block interference (IBI) and reduce receiver complexity. Although OFDM is very effective in combating the effect of delay-spread, it stills suffers from signal fading caused by multipath propagation as well as interference caused by other users. Receiver diversity [2] is a technique for mitigating both effects. For example, by linearly combining the signals from multiple receiving antennas, a diversity receiver can greatly enhance the performance of an OFDM system in the presence of signal fading and interference. The weightings for diversity combining must be carefully optimized in order to achieve the best performance, which in turns requires accurate estimates of the channel between all users and receiver antennas.

The development of blind channel estimation and equalization algorithms based on second-order statistics (SOS) [3] derived either by over-sampling or from the use of an antenna array has spawned intensive research in the area, such as the least-squares [12] and

This research work was supported by the National Science Council of the Republic of China under grant number NSC 90-2219-E-002-011. the subspace [8] methods. The subspace method, in particular, has also been applied to channel estimation for single-user OFDM systems [4-6]. A subspace-based approach is proposed in [4] to directly estimate the frequency-domain channel gains for single-user OFDM systems. In [5], a subspace-based channel identification algorithm is proposed for a single-user OFDM system without cyclic prefix. And in [6] it is proposed to use the autocorrelation of the vector that consists of all received signal samples to perform subspace-based blind channel estimation for single-user OFDM systems. While these approaches achieve good performance for the single-user case, some generalizations are necessary if they are to be applied to multi-user OFDM systems.

In this paper, we present a receiver that uses the subspace-based channel estimation algorithm for multi-user OFDM transmission systems with receiver diversity. The proposed receiver consists of a two-step blind channel estimator and a frequency-domain linear diversity combiner. In the channel estimator, an initial time-domain channel estimate is first obtained using a new multi-user generalization of the subspace approach. The initial estimate is then transformed into the frequency domain and appropriately scaled to obtain the refined, final frequency-domain channel estimates. The diversity combiner then performs per-subcarrier linear combining using weights computed from the frequency-domain channel estimates. Simulation results show that performance gain of $1-3 \mathrm{~dB}$ is achievable using the proposed approach.

\section{SySTEM BLOCK DIAGRAM}

Consider the multi-user OFDM transmission system shown in Fig. 1, in which $S$ quasi-synchronous users transmit independent and identically distributed OFDM symbols in the same bandwidth at the same time. For the $s$-th user, information bits are convolutionally encoded and mapped onto quaternary phase-shift keying (QPSK) 
subsymbols, which are then serial-to-parallel converted and processed by the $N$-point inverse discrete Fourier transformer (IDFT), where $N$ is the number of subcarriers. The output of IDFT is parallel-to-serial converted and a CP of length $N_{C P}$ is inserted to obtain a discrete-time signal $x_{s, l}$. For simplicity, we assume that CP is sufficiently long so that there is no IBI. The continuous-time transmitted signal of user $s$ is given by

$$
x_{s}(t) \equiv \sum_{l} x_{s, l} q(t-l T),
$$

where $T$ is the sample (chip) time of the OFDM symbol, and $q(\bullet)$ is the pulse-shaping function. The user signals are transmitted to $M$ receiving antennas through independent wireless channels, each modeled as a modified Jakes' frequency-selective multipath fading channel corrupted by additive white Gaussian noise (AWGN) [7].

At the receiver, the signals $u^{(m)}(t)$ received from the $m$-th antenna $(m=0, \ldots, M-$ 1) are each filtered by a lowpass filter and sampled at a rate of $P / T$ samples per second, resulting in a discrete-time signal given by

$u_{n, p}^{(m)} \equiv u^{(m)}\left(n T+\frac{p T}{P}\right)=\sum_{s=0}^{S-1} \sum_{b=0}^{L-1} x_{s, n-b} h_{b, s, p}^{(m)}+v_{n, p}^{(m)}$

for $m=0,1, \ldots, M-1, p=0,1, \ldots, P-1$, and $n=0,1$, $2, \ldots$, where $h$ is the poly-phase discrete-time equivalent channel and $L$ is the channel memory. The signal $u_{n, p}^{(m)}$ is then fed into the proposed receiver to be discussed in the next section.

\section{Proposed RECEIVER AlgORITHM}

As shown in Fig. 1, the proposed receiver consists of a two-step blind channel estimator and a frequency-domain linear diversity combiner. The two-step blind channel estimator produces an accurate estimate of the poly-phase discrete-time equivalent channel. The linear diversity combiner combines the frequency-domain received signals of all antenna elements on a per-subcarrier basis using weightings derived from the channel estimates using the minimum mean-square error (MMSE) criterion. The output is finally detected and decoded to obtain the decoded information bits.

\section{A. Two-Step Blind Channel Estimation}

The proposed blind channel estimation algorithm consists of two steps. In the first step, an initial time-domain channel estimate is obtained using a new multi-user generalization of the subspace approach proposed in [8]. In the second step, a refined frequency domain channel estimate is obtained by appropriately transforming and scaling the initial channel estimate. The resulting channel estimation algorithm is referred to as the subspace-MMSE channel estimates. Mathematically, the input to the blind channel estimator is an $N P$-dimensional poly-phase vector given by

$$
\mathbf{u}^{(m)} \equiv\left[\begin{array}{lll}
\mathbf{u}_{0}^{(m) T} & \cdots & \mathbf{u}_{P-1}^{(m) T}
\end{array}\right]^{T},
$$

where

$$
\mathbf{u}_{p}^{(m)}=\left[\begin{array}{lll}
u_{n, p}^{(m)} & \cdots & u_{n-N+1, p}^{(m)}
\end{array}\right]^{T}
$$

In this paper, the $N$ components in $\mathbf{u}_{p}^{(m)}$ are assumed to be samples of a particular OFDM symbol after the CP is discarded. Let $\mathbf{R}_{\mathbf{u}}^{(m)}$ be the estimated covariance matrix of $\mathbf{u}^{(m)}$, and let $\mathbf{n}_{i}^{(m)}, i=1,2, \ldots, N P-S(L+N)$ be the eigenvectors corresponding to the smallest eigenvalues of $\mathbf{R}_{\mathbf{u}}^{(m)}$. Following the derivations in [8], initial estimates $\tilde{h}_{b, s, p}^{(m)}$ of $h_{b, s, p}^{(m)}$ that are accurate to within a scaling constant are obtained by minimizing $\widetilde{\mathbf{h}}_{s}^{(m) H} \mathbf{Q}^{(m)} \widetilde{\mathbf{h}}_{s}^{(m)}$ subject to $\mathbf{C}^{H} \widetilde{\mathbf{h}}_{s}^{(m)}=1$ (linear constraint) or $\left\|\widetilde{\mathbf{h}}_{s}^{(m)}\right\|=1$ (quadratic constraint), where $\mathbf{C}$ is an arbitrary $P L$-dimensional vector,

$$
\widetilde{\mathbf{h}}_{s}^{(m)}=\left[\begin{array}{lll}
\widetilde{\mathbf{h}}_{s, 0}^{(m)} & \cdots & \widetilde{\mathbf{h}}_{s, P-1}^{(m)}
\end{array}\right]^{T}
$$

is the initial time-domain estimate of the poly-phase discrete-time equivalent channel response between the $s$-th user and the $m$-th antenna, in which

$$
\tilde{\mathbf{h}}_{s, p}^{(m)}=\left[\begin{array}{lll}
\tilde{h}_{0, s, p}^{(m)} & \cdots & \tilde{h}_{L-1, s, p}^{(m)}
\end{array}\right],
$$

and $\mathbf{Q}^{(m)}$ is a matrix function [8] of $\mathbf{n}_{i}^{(m)}, i=$ $1,2, \ldots, N P-S(L+N)$.

After the initial time-domain channel estimates are obtained, refined frequency-domain channel estimates are obtained by first taking the DFT of $\mathbf{u}_{p}^{(m)}$ to obtain $\mathbf{U}_{p}^{(m)}$. Let

$$
\mathbf{U}^{(m)}=\left[\begin{array}{llll}
\mathbf{U}_{0}^{(m) T} & \mathbf{U}_{1}^{(m) T} & \ldots & \mathbf{U}_{P-1}^{(m) T}
\end{array}\right]^{T},
$$

we have

$$
\mathbf{U}^{(m)}=\mathbf{H}^{(m)} \mathbf{X}+\mathbf{V}^{(m)},
$$

where

$$
\mathbf{X}=\left[\begin{array}{lll}
\mathbf{X}_{0}^{T} & \cdots & \mathbf{X}_{S-1}^{T}
\end{array}\right]^{T},
$$

in which

$$
\mathbf{X}_{s}=\left[\begin{array}{lll}
X_{0, s} & \cdots & X_{N-1, s}
\end{array}\right]^{T}
$$

is the vector of transmitted subsymbols of the $s$-th user and $\mathbf{V}^{(m)}$ is a Gaussian random vector. The matrix $\mathbf{H}^{(m)}$ is the multi-user poly-phase frequency-domain channel matrix of the $m$-th antenna that has $P$ block rows and $S$ block 
columns. The $(p, s)$-th block element of $\mathbf{H}^{(m)}$ is an $N \times N$ diagonal matrix whose diagonal entries are the DFT values of $\mathbf{h}_{s, p}^{(m)}$.

As mentioned earlier, suitable scaling factors are necessary for obtaining the final frequency-domain channel estimates. Let

$$
\hat{H}_{k, s, p}^{(m)}=C_{k, s}^{(m)} \widetilde{H}_{k, s, p}^{(m)}
$$

be the final frequency-domain channel estimate, where $\widetilde{H}_{k, s, p}^{(m)}, k=0, \ldots, N-1$, are the initial frequency-domain channel estimates obtained by taking the DFT of $\widetilde{\mathbf{h}}_{s, p}^{(m)}$, and $C_{k, s}^{(m)}$ are the appropriate scaling factors to be determined. Define the mean-square error (MSE) as

$$
J^{(m)} \equiv E\left(\left\|\mathbf{U}^{(m)}-\hat{\mathbf{H}}^{(m)} \mathbf{X}\right\|^{2}\right)
$$

where $\hat{\mathbf{H}}^{(m)}$ is an estimate of $\mathbf{H}^{(m)}$ obtained using by replacing the entries of $\mathbf{H}^{(m)}$ with $\hat{H}_{k, s, p}^{(m)}$. Substituting (11) into (12), we have

$$
J^{(m)}=E\left(\left\|\mathbf{U}_{k}^{(m)}-\widetilde{\mathbf{H}}^{(m)} \boldsymbol{\alpha}^{(m)}\right\|^{2}\right)
$$

where

$$
\boldsymbol{\alpha}^{(m)} \equiv\left[\begin{array}{lll}
\boldsymbol{\alpha}_{0}^{(m) T} & \cdots & \boldsymbol{\alpha}_{S-1}^{(m) T}
\end{array}\right]^{T}
$$

in which

$\boldsymbol{\alpha}_{s}^{(m)} \equiv\left[\begin{array}{lll}C_{0, s}^{(m)} X_{0, s}^{(m)} & \cdots & C_{N-1, s}^{(m)} X_{N-1, s}^{(m)}\end{array}\right]^{T}$

The scaling factors $C_{k, s}^{(m)}$ are found by first computing the MMSE solution for $\boldsymbol{\alpha}^{(m)}$ given by [9]

$$
\boldsymbol{\alpha}^{(m)}=\left(\widetilde{\mathbf{R}}^{(m)}\right)^{-1} \widetilde{\mathbf{p}}^{(m)}
$$

where

$$
\widetilde{\mathbf{R}}^{(m)} \equiv E\left(\widetilde{\mathbf{H}}^{(m) H} \widetilde{\mathbf{H}}^{(m)}\right)
$$

and

$$
\widetilde{\mathbf{p}}^{(m)} \equiv E\left(\widetilde{\mathbf{H}}^{(m) H} \mathbf{U}^{(m)}\right) .
$$

The scaling factors are then given by

$$
C_{k, s}^{(m)} \equiv \frac{1}{\sigma_{X}}\left|\alpha_{k, s}^{(m)}\right|
$$

where $\sigma_{X}^{2}$ is the average energy of the QPSK subsymbols. $\alpha_{k, s}^{(m)}$ are the components of $\boldsymbol{\alpha}_{s}{ }^{(\mathrm{m})}$. Note that in practice, the statistical expectations in (17) and (18) can be replaced by averages over several OFDM symbols.

Note that in the proposed receiver, the channels are separately estimated for each antenna using over-sampled received signals. When $M>S$, it is straightforward to extend the proposed algorithm so that all channels are jointly estimated using chip-spaced $(P=1)$ received signal samples ${ }^{1}$.

\footnotetext{
1 Joint estimation using over-sampled received signal is not considered in this paper due to its high complexity.
}

B. Frequency-Domain Linear Diversity Combining

After the final channel estimates are obtained, the received signals from each antenna are linearly combined in the frequency domain on a per-subcarrier basis in order to combat signal fading and cancel interference. The weightings for linear combination are obtained based on the final channel estimates and the MMSE criterion. Mathematically, assuming that user 0 is the desired user, the output of the linear combiner is an $\mathrm{N}$-dimensional vector estimate of $\mathbf{X}_{0}$ that is given by

$$
\hat{\mathbf{X}}_{0}=\mathbf{W}_{0}^{H} \mathbf{U},
$$

where

$$
\mathbf{U}=\left[\begin{array}{lll}
\mathbf{U}^{(0) T} & \cdots & \mathbf{U}^{(M-1) T}
\end{array}\right]^{T},
$$

and $\mathbf{W}_{0}$ is an $M N P \times N$ weighting matrix that minimizes the MSE of each component of $\hat{\mathbf{X}}_{0}$. It can be shown that $\mathbf{W}_{0}=\mathbf{R}^{-1} \mathbf{P}$, where

$$
\mathbf{R}=E\left[\mathbf{U U}^{H}\right]
$$

and $\mathbf{P}$ is an $M N P \times N$ matrix obtained by stacking the first block column of $\hat{\mathbf{H}}^{(0)}$ to $\hat{\mathbf{H}}^{(M-1)}$.

\section{SimULATION RESULTS}

The performance of the proposed receiver algorithms is evaluated using computer simulation. User information bits are first encoded using a convolutional encoder and mapped onto $N=64$ QPSK subsymbols. $N_{C P}$ is set to 16 and a $35 \%$ roll-off square-root raised cosine function is used for pulse shaping. The wireless channels have power-delay profiles following the Typical Urban (TU) channel models [10]. The receiver filters used in the simulations are also $35 \%$ roll-off square-root raised cosine filters. The carrier frequency is assumed to be $900 \mathrm{MHz}$. No frequency offset is assumed between the transmitter and the receiver. The maximum normalized Doppler frequency (defined as the maximum Doppler frequency normalized by the OFDM symbol period) is 0.025 , which corresponds a vehicle speed of $40 \mathrm{~km} / \mathrm{h}$. Unless otherwise stated, the number of active users is fixed at $S=2$.

The normalized mean-square error (NMSE), defined as

$$
N M S E \equiv \frac{\sum_{m=0}^{M-1} \sum_{s=0}^{S-1} \sum_{p=0}^{P-1} \sum_{k=0}^{L-1}\left|\hat{H}_{k, s, p}^{(m)}-H_{k, s, p}^{(m)}\right|^{2}}{\sum_{m=0}^{M-1} \sum_{s=0}^{S-1} \sum_{p=0}^{P-1} \sum_{k=0}^{L-1}\left|H_{k, s, p}^{(m)}\right|^{2}}
$$

of the proposed channel estimation algorithm and previous channel estimation algorithms are 
shown in Fig. 2 for the TU channel with as functions of the number of observation symbols. Here the signal-to-interference ratio (SIR) is 10 $\mathrm{dB}$ and $E_{b} / N_{0}$ is $20 \mathrm{~dB}$, where $E_{b}$ is the energy per transmitted bit and $N_{0} / 2$ is the two-sided power spectral density of the AWGN. The over-sampling factor $P=4$ and number of receiver antennas $M=2$. It can be seen from Fig. 2 that the proposed method requires 100 OFDM symbols to achieve $\mathrm{NMSE}<0.1$, while the conventional subspace approach with linear and quadratic constratins [8] need about 250 and 200 OFDM symbols, respectively.

The decoded bit error rate (BER) of the proposed receiver with over-sampling factor $P$ $=4$ and number of receiver antennas $M=2$ is shown in Fig. 3(a) as functions of $E_{b} / N_{0}$ and Fig. 3(b) as functions of SIR for the TU channel. In Fig. 3(a) SIR is fixed at $4 \mathrm{~dB}$ and in Fig. 3(b) $E_{b} / N_{0}$ is fixed at $30 \mathrm{~dB}$. The performance of linear diversity combiners using the "perfect" channel estimates as well as channel estimates obtained using the conventional subspace approach with linear and quadratic constraints are also shown in this figure as baselines for comparison. It can be seen that the proposed receiver outperforms the conventional subspace approaches by 1 to $3 \mathrm{~dB}$. This is because in the proposed approach, the initial channel estimates obtained using the subspace approach are appropriately scaled before being used to compute the weights for diversity combining.

In Fig. 4, the decoded BER of the proposed receiver with $M=3$ is shown as functions of (a) $E_{b} / N_{0}$ and (b) SIR for the TU channel. An additional curve labeled as "joint channel estimation" is also shown in the figures. This curve is obtained by letting $P=1$ and jointly estimating all channels as mentioned in Section III. Comparing Fig. 4 to Fig. 3, it can be seen that increasing the number of antennas from $M=2$ to $M=3$ achieves significant performance gain as expected. Furthermore, it can also be seen that when $M>S$, joint estimation can be performed instead of over-sampling, although separately estimating the channels for each antenna using $P=4$ achieves better performance.

\section{CONCLUSION}

A receiver algorithm for OFDM with receiver diversity is proposed in this paper. The proposed receiver consists of the two-step subspace-MMSE blind channel estimator and a linear frequency-domain diversity combiner. The first step of the subspace-MMSE channel estimator uses a new multi-user generalization of the subspace-based blind channel estimator to obtain an initial time-domain channel estimate. In the second step, the initial channel estimates are appropriately transformed and scaled to obtain the refined final frequency-domain channel estimates. The linear diversity combiner then computes the optimal (MMSE) combination weightings using the refined channel estimates. Simulation results show that performance gains of $1-3 \mathrm{~dB}$ is achievable over conventional subspace channel estimators.

\section{REFERENCES}

[1] R.V. Nee and R. Prasad, OFDM for Wireless Multimedia Communications, Artech House, 2000.

[2] J. C. Liberti and T. S. Rappaport, Smart Antennas for Wireless Communications: IS-95 and Third Generation CDMA Application, Prentice Hall, 1999.

[3] L.Tong, G. Xu, and T. Kailaith, " Fast blind equalization via antenna arrays," ICASSP-93, vol.4, pp. 272-275

[4] B. Muquet, M. de Courville, P. Duhamel and V. Buzenac, "A subspace based blind and semi-blind channel identification method for OFDM systems," SPAWC '99, pp. 170-173

[5]C. Li and S. Roy, “A subspace blind channel estimation method for OFDM systems without cyclic prefix," VTC 2001 Fall, vol.4, pp.2148-2152

[6]X. Cai and A.N. Akansu, "A subspace method for blind channel identification in OFDM systems," ICC 2000, vol. 2 , pp. $929-933$

[7] P. Dent, G.E. Bottomley, and T. Croft, "Jakes fading model revisited," Electronics Letters, vol.29, no.13, pp.1162-1163, June 1993.

[8] E. Moulines, P. Duhamel, J. F. Cardoso, and S. Mayrargue, "Subspace methods for the blind identification of multichannel FIR filters," IEEE Trans. Signal Processing, vol.43, no.2, Feb. 1995, pp. 516-525

[9] A. Papoulis, Probability, Random Variables, and Stochastic Processes, 3rd Ed., McGraw-Hill, 1991.

[10] 3G TR 25.943: 3rd Generation Partnership Project; Technical Specification Group(TSG) RAN WG4; Deployment aspects.

[11] G. Xu, H. Liu, L. Tong, and T. Kailath, “A Least-Squares Approach to Blind Channel Identification," IEEE Trans. On Signal Processing, Vol. 43, pp. 2982 - 2993, December 1995. 


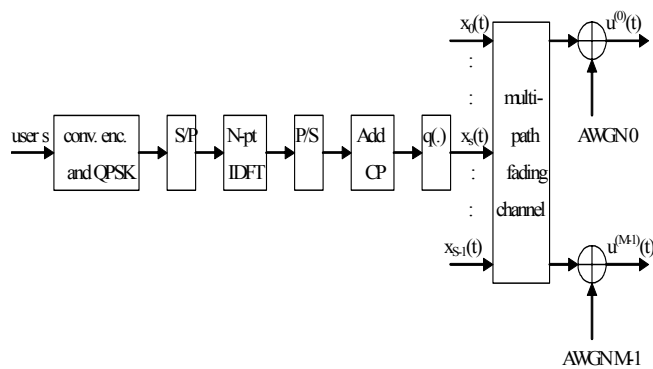

Transmitter and channel

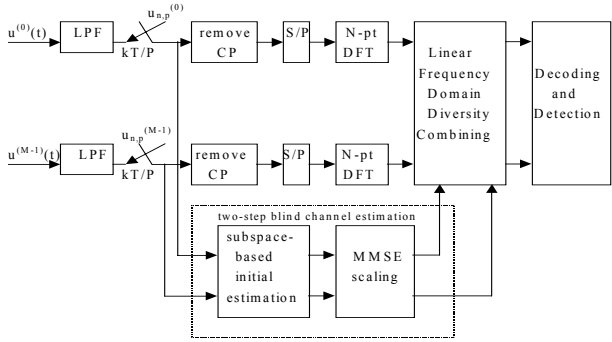

Receiver

Fig.1. A multi-user OFDM transmission system with receiver diversity.

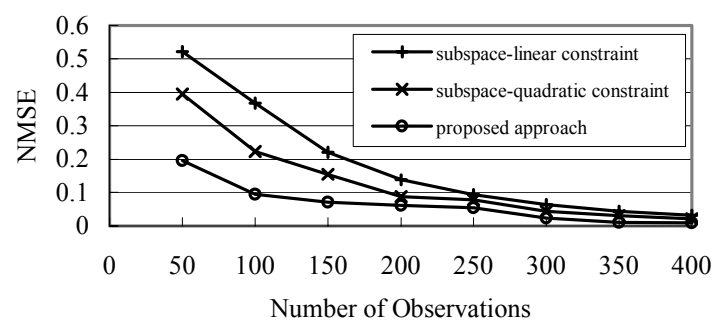

Fig.2. The normalized mean square error (NMSE) as functions of the number of observation symbols.

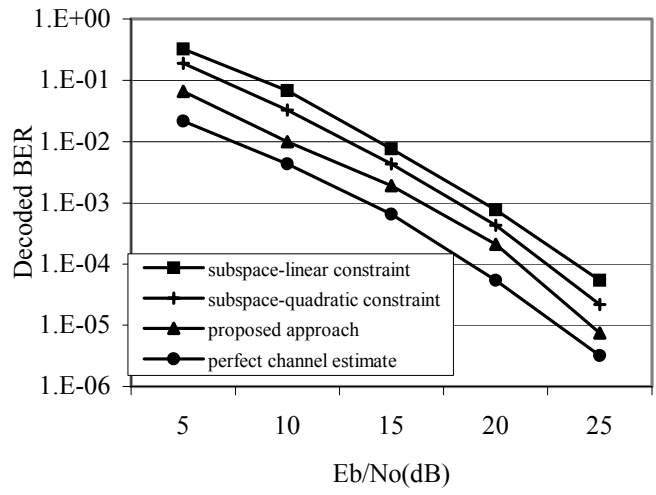

(a)

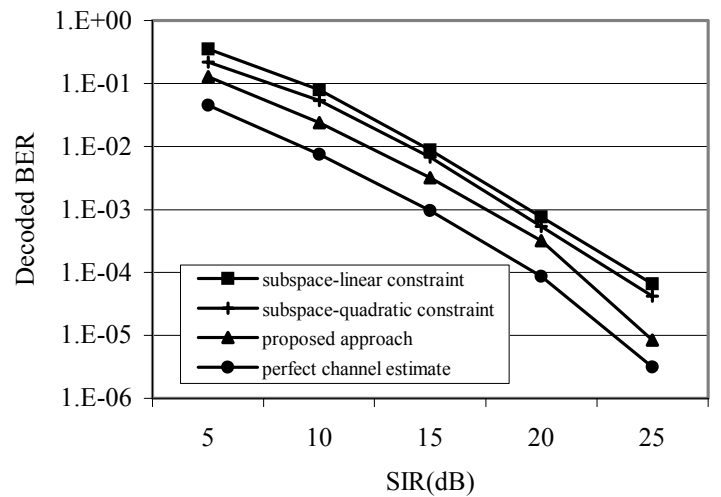

(b)

Fig.3 (a) BER versus $E_{b} / N_{0}$ and (b) BER versus SIR for TU channel.

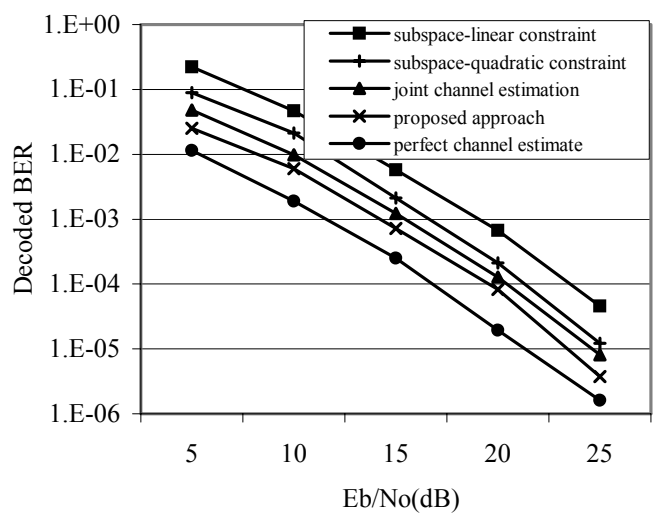

(a)

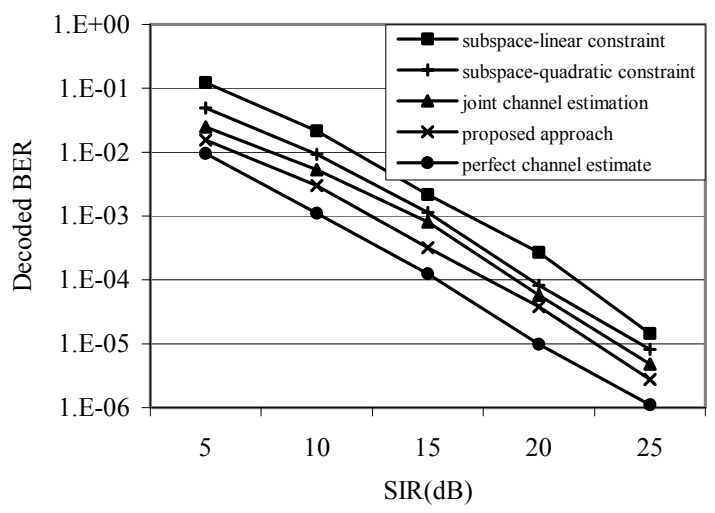

(b)

Fig.4. Comparison of the system performance for different channel estimation algorithms when $M=3$ :

(a) BER versus $E_{b} / N_{0}$ (b) BER versus SIR 